\title{
Role of miRNA manipulation on methylation states of breast cancer stem cell-related gene: Methylation status and miRNAs on BCSCs
}

\author{
Heba H. El-Osaily ${ }^{1, *}$, Iman H. Ibrahim ${ }^{2}$, Mona L. Essawi ${ }^{3}$, Sohair M. Salem ${ }^{4}$ \\ ${ }^{1}$ Department of Biochemistry, Faculty of Pharmacy, Ahram Canadian University, Giza 12585, Egypt. \\ ${ }^{2}$ Department of Biochemistry, Faculty of Pharmacy (Girls), Al-Azhar University, Cairo 11651, Egypt. \\ ${ }^{3}$ Department of Medical Molecular Genetics, National Research Centre, Giza 12622, Egypt. \\ ${ }^{4}$ Department of Molecular Genetics and Enzymology, National Research Centre, Giza 12622, Egypt.
}

${ }^{*}$ Corresponding author: E-mail: hebaelosaily2@gmail.com, Ahram Canadian University, $4^{\text {th }}$ industrial region, $6^{\text {th }}$ October, Egypt. Tel.: +201221620730; Fax: +2(02) 38334379, ORCID: 0000-0003-3244-1479.

Article history: Received 2021-02-05

Revised 2021-03-15

Accepted 2021-04-30

Abstract: The genetic and epigenetic alteration was commonly related to the progression of breast cancer. Epigenetic alteration which comprises DNA methylation and microRNA is involved in controlling the gene expression which is related to cancer cells. The change of cellular transformation, tumorigenesis, and stemness marker were accomplished by this epigenetic modification. The short, non-coding RNAs, which are called microRNAs (miRNAs) are considered as a master regulator of genes and are associated with the management of both physiological and pathological status. The abnormal miRNAs expression was found to be contributed in the progress of many types of human tumors by disrupting the action of oncogenic and cancer suppressor genes. For the meantime, the expression of both oncogenic and tumor suppressor genes was affected by the change in the DNA methylation status. This occurs due to the hypermethylation of CpG islands within the promoter sites, that disturbs the tumor suppressing role of miRNAs in cancer. The current article will talk about the relationship of miRNAs and DNA methylation and its effect on the adjustment of gene expression.

Key Words: mi-RNA; Breast Cancer; Epigenetics; Stem Cells; Methylation.

\section{INTRODUCTION}

The first known cancer stem cells (CSCs) were breast cancer stem cells (BCSCs) 1. The presence of CD44+ /CD24- or aldehyde dehydrogenase-1 (ALDH1) phenotypes were found to be the hallmark for differentiation between BCSCs and tumor samples. CSCs have a critical role in cancer progression and resistance to therapy in breast cancer (BC). Therefore, BCSCs targeting has the ability to enhance the recovery for women suffering from BC 1. Liu et al, (2014) documented that at the mesenchymal to epithelial state (MET), the ALDH+ BCSCS were existed and concentrated at the interior of the tumor and are mostly proliferative 2 . While at the epithelial to mesenchymal (EMT) the CD44+/CD24- BCSCs were existed and concentrated at the invasive edge of the tumor and are mainly quiescent. Their results counsel that BCSCs either in EMT or MET state might have a diverse effect on tumor progression and invasion $^{3}$ (Figure1).
Morphological changes like cytoskeletal rearrangement, polarity loss, and cell-cell contact were performed to epithelial-like stem cells to become mesenchymal-like stem cells during the $\mathrm{EMT}^{4}$.

The BCSCS have their role in the tumor prognosis phenotype progression in addition to invasion. As mentioned before BCSCs transition from epithelial-like to mesenchymal-like is facilitated by stromal cell interaction and improve the invasion of BCSCS. Tumors enriched with CSCs and BCSCs were showed poor prognosis phenotype due to their high phenotypic plasticity ${ }^{6}$. The most vital regulators for gene expression are DNA methylation and miRNA. The development of the pathological condition is due to the alteration in miRNA expression or DNA methylation. miRNA transcription can be inhibited by methylation of the promoter region which includes $\mathrm{CpG}$ islands. In contrast, the action of DNA methyltransferase could be suppressed by direct miRNA targeting, which affects the methylation pattern of the whole genome.

Cite this article: Elosaily, H., Ibrahim, I., Essawi, M., Salem, S. Role of miRNA manipulation on methylation states of breast cancer stem cell-related gene: Methylation status and miRNAs on BCSCs. Azhar International Journal of Pharmaceutical and Medical Sciences, 2021; 1(2):13-22.

DOI: 10.21608/aijpms.2021.61532.1045 
This review put the spot on the link between DNA methylation and miRNA at protein and gene expression level ${ }^{7}$.

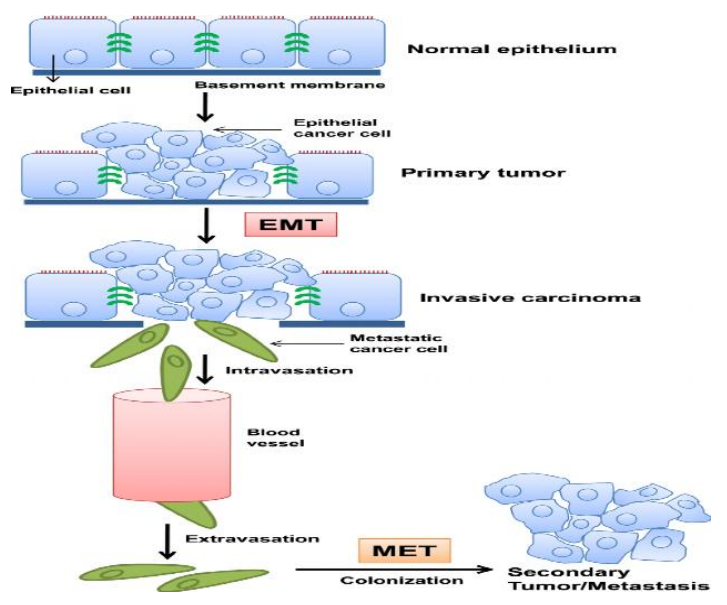

Figure (1): The reversible process of EMT at $\mathrm{CSC}^{5}$.

\section{CANCER STEM CELLS MARKERS}

The BCSCs subpopulation could be identified by different set of markers. Surface markers have been used to identify and isolate BCSCs and give them a unique character ${ }^{8}$.

\subsection{CD44}

The transmembrane glycoprotein (CD44) is considered as a promoting factor in the development of different types of cancer. The abnormal expression of CD44 was contributed to the aggressiveness and initiation of BC. Also, CD44 serves as marker for CSC which stimulates invasion and metastasis of tumor as it involved in the EMT process ${ }^{9}$.

\subsection{CD24}

The mucin-like surface protein is known as CD24 contains glycosylation sites that adhere to Pselectin ${ }^{10}$. CD24 acts as an adhesion marker so, the lowered expression level of CD24 rises the ability of the tumor to invade ${ }^{11}$. Some reports documented that $\mathrm{CD} 24$ has a role in the EMT-MET transition in of $\mathrm{BC}^{12}$.

The tumorigenic phenotype of the CD $44^{+} / \mathrm{CD} 24^{-}$ subpopulation was verified to be associated with stem cell-like character. This subpopulation is the most used marker to define BCSCs as it was first predicted in $2003{ }^{13}$. Consequently, CD44 together with CD24 was considered as a marker for stemness in $\mathrm{BC}{ }^{14}$.

\subsection{ALDH1A}

ALDHs are a group of NADP+ dependent enzymes. This enzyme oxidized the aldehyde substrates to carboxylic acids exogenously and endogenously. The activity of ALDHs was increased in tumor cells and act as a potential prognostic marker of $\mathrm{CSCs}^{12}$.

\subsection{NANOG, OCT3/4 and SOX2}

The main regulator of pluripotency is OCT3/4, SOX2, and NANOG. These transcription factors were worked together to keep cell undifferentiated ${ }^{15}$. The expression of OCT3/4and SOX2 in different tumor tissue but not normal tissue was showed poor prognosis of some tumors ${ }^{16}$. Also, NANOG and OCT3/4 were found to be highly expressed in tumor cells but absent or slightly expressed in the cell with mature organization. Notably, the aggressiveness of the tumor is positively linked to the expression level of these transcription factors ${ }^{17}$.

One of the POU domain family members is the octamer binding transcription factor (OCT3/4). OCT3/4 is expressed in diverse types of stem cells such as embryonic stem cell, germ cell, and adult human cell. OCT3/4 has been contributed to the invasiveness and self-renewal properties of ESC. OCT3/4 was expressed in several types of cancer and contributed to the development of tumor ${ }^{18}$.

SOX2 is the sex-determining region Y-box 2 of the SOX family that is belonging to group B. It is a significant transcription factor in keeping the recurrence properties of ESCs and neural progenitor cells. It was found that SOX2 is expressed in high levels in tumor cells and responsible for the differentiation and progression of cancerous cells. SOX2 and OCT3/4 act together to regulate the DNA transcription and play a significant role in the controlling of gene expression ${ }^{19}$.

The key transcription factor homeobox protein (NANOG) comprises of 305 amino acid, the selfrenewal property of ESC was maintained by its effect 20. NANOG works in conjugation with SOX2 and OCT3/4 to form the identity of ESC. It is also overexpressed in different types of cancer including BC. Also, NANOG forms the core of the transcription network with SOX2 ${ }^{21}$.

We revealed protein-protein interaction in $\mathrm{BC}$ by STRING platform. Possible interactions of OCT3/4, SOX2, and NANOG with other genes were found as showed in (Figure 2). Several aspects displayed Protein-protein interactions with SOX2, NANOG, and OCT3/4 were predicted using STRING database. From (Figure 2) we can find that KLF4, CTNNB1, SALL4, DPPA4, LIN28A, TDGF1, and STAT4 were showed to have protein interactions with SOX2, NANOG, and OCT3/4.

Several aspects displayed Protein-protein interactions with SOX2, NANOG, and OCT3/4 were predicted using STRING database. From (Figure 2) we can find that KLF4, CTNNB1, SALL4, DPPA4, LIN28A, TDGF1, and STAT4 were showed to have 
protein interactions with SOX2, NANOG, and OCT3/4.

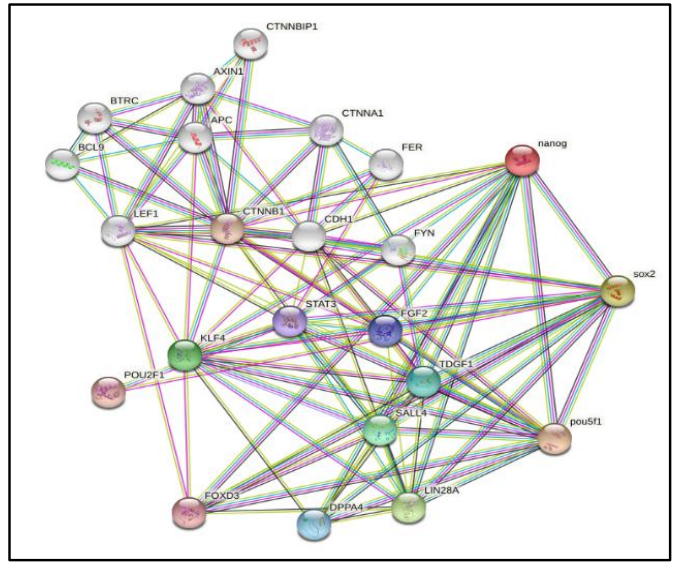

Figure (2): Network of Protein interaction by the STRING platform of OCT3/4, SOX2, and NANOG. Each colored line between proteins indicates diverse types of interaction. The indication for these interactions was resulting from the database (blue line), and co-expression (black line) ${ }^{22}$.

According to this protein interaction network, each gene has a role in cancer prognosis and tumor formation. TDGF-1 controls the development of ESC and its differentiation. In normal cells, TDGF-1 was found to be expressed at a decreased level while it is expressed at high levels in cancerous tissue. It has a role in tumor metastasis ${ }^{23}$. As well, Dppa4 was found to have increased expression in many cancerous cells and was also considered as a definite pluripotent cell marker ${ }^{24 .}$

Zinc-finger transcription factor (Klf4) is overexpressed in $\mathrm{BC}$ and performed as an oncogene while, it plays a role in the proliferation of cells ${ }^{25}$. Meanwhile the action of FGF2 on the ER and PR enhances the tumor proliferation and growth ${ }^{26}$.

On the other hand, SALL4 and CTNNB1 act together to enhance the activity of the $\mathrm{Wnt} / \beta$-catenin signaling pathway which increases the rate of tumor formation ${ }^{27}$. The abnormal expression of LIN28A is linked to cancer progression in many types of cancer and acts as a posttranscriptional regulator of a gene involved in self-renewal of ESCs ${ }^{22}$. While, STAT3 was used as an early indicative marker and is recognized to initiate the tumorgenicity of the $\mathrm{BC}^{28}$.

\section{EPIGENETICS}

The process which regulates the expression of genes without affecting the sequence of DNA is known as epigenetic ${ }^{29}$. The epigenetic alterations have the ability to change the expression of a gene by multiple ways; involving methylation of DNA, histone modifications, and miRNA. These three mechanisms are the main

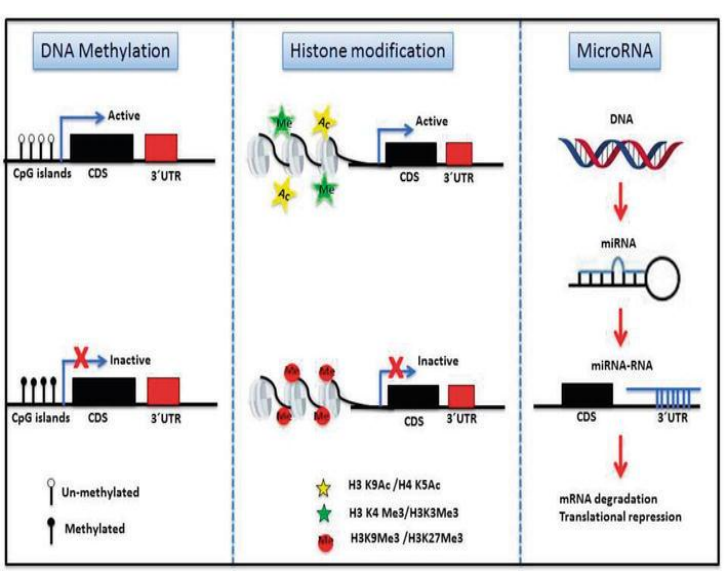

Figure (3): The Epigenetic mechanisms affecting gene expression ${ }^{30}$.

epigenetic modification which has an important role in altering the gene expression as shown in (Figure 3).

In human, the most known epigenetic modification is DNA methylation. The DNA methyltransferase (DNMT) is catalyzing the reaction by adding a methyl group to the 5 position of the pyrimidine ring of a cysteine nucleotide. The types of DNA methyltransferase enzymes are DNMT1, DNMT3A, and DNMT3B. DNMT enzymes control gene methylation pattern. DNMT enzyme regulate properties of stem cell by de novo methylation 31 .

Through the human genome, $\mathrm{CpG}$ dinucleotides are widely distributed and nearly occur in the frequency of one for every 80 dinucleotides. CpGs island methylation is the most common form of methylation. The human genome contains $\mathrm{CpG}$ island which has a high content of $\mathrm{CpG}$ dinucleotide. These regions are frequently located in the promoter region $^{32}$.

Genes which are transcribed in the normal range have unmethylated promoter region. Hypermethylation of promoter region leads to gene inactivation while the decreasing in methylation level leads to gene activation. Promoter methylation can cause gene deactivation by several mechanisms ${ }^{33}$. Blocking the binding site of a transcription factor by methylation is considered one of these mechanisms. The other mechanism concluded that methylation blocks the entrance of factors needed for gene expression ${ }^{34}$. Moreover, DNA methylation could be contributed in condensed packing of chromatin resulting in inactive regions (heterochromatin). Methylation of promoter regions is contributed to the deactivation of gene, while the activation of genes is occurred by gene body methylation ${ }^{35}$. 


\subsection{DNA Methylation and Cancer}

Abnormalities like methylation loss (hypomethylation) and gain (hypermethylation), are related to cancer progression. The global hypomethylation and the promoter hypermethylation of genes which are accounted as tumor suppressor are usually detected in cancer ${ }^{36}$. Tumor suppressor genes can be deactivated by either hypermethylation of the promoter region or mutation. The abnormal increase of methylation in the promoter region of tumor suppressor genes has been reported in $\mathrm{BC}$ such as CDH1, RASSF1A, and BRCA1 ${ }^{37}$.

Genome wide DNA hypomethylation is the second most vital modification in DNA methylation. The DNA hypomethylation of repetitive sequence including short interspersed transposable element (SINE) and long interspersed transposable element (LINE) causes chromosomal reorganization and defect in cells that leads to genetic instability. Chromosomal rearrangement associated with the enhancement of cancer growth as shown in (Figure 4).

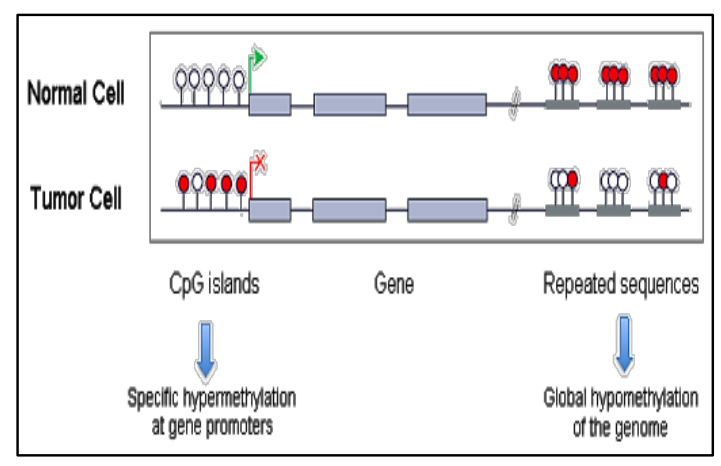

Figure (4): Abnormal DNA methylation in cancer cells ${ }^{36}$.

The global genomic methylation status can be distinguished by the methylation level of repetitive transposable DNA elements. Accumulating evidence presented that the repetitive DNA elements located at high frequency in intronic regions of the genome ${ }^{38}$. While the $\mathrm{CpG}$ sites are frequently methylated inside the repetitive DNA elements. LINE-1 is the longinterspersed nucleotide element- 1 and is considered as a major component of repetitive transposable DNA elements, as it makes up about $17 \%$ of the human genome ${ }^{39}$.

LINE-1 is regularly methylated in normal cells. The methylation of LINE-1 deactivates the transcription and prevents retro transposition. It has been documented that the degree of methylation of LINE-1 is lowered in many types of cancer tissues when compared to normal ones ${ }^{40}$.

\subsubsection{The effect of DNA methylation on} Breast Cancer Stem Cells

The regulation of BCSC genes is controlled by the methylation level of $\mathrm{CpG}$ island in the promoter region. The alteration of the methylation level could stimulate BCSCs gene this may lead to an increase in the aggressiveness of $\mathrm{BC}$ phenotype ${ }^{41}$. Moreover, the progression of $\mathrm{BC}$ can be influenced by DNA hypermethylation as it decreases the tumor suppressor genes, and DNA hypomethylation as it over expresses oncogenes. In fact, that DNA hypermethylation is depressingly correlated with gene expression ${ }^{42}$.

As mentioned before OCT3/4 is important for maintaining the ESC. It also, plays a role in metastasis and development of cancer ${ }^{22}$. The over expression of OCT3/4 leads to tumor progression. Also, the expression of DNMT1 and DNMT3B contributes to carcinogenesis. DNA methylation regulates the expression of OCT3/4 in ESC ${ }^{43}$. Hence, there is a correlation between OCT3/4 and DNA methyl transferases enzyme expression. DNMTs expression can be enhanced by the co-expression of OCT3/4/SOX2 ${ }^{44}$. Also, Transcription factor and miRNA which act as epigenetic regulators, enhancing the CSCs transition ${ }^{45}$.

\subsection{MicroRNA}

MicroRNAs (miRNA) are small single stranded, non-coding RNAs contain approximately $\sim 22$ nucleotides. The endogenously expressed miRNA controls the expression of genes by joining to 3'untranslated region (3'-UTR) in their mRNA target ${ }^{46}$. This regulation affects the protein translation either by degradation or repression ${ }^{47}$. Biological processes such as differentiation, metastasis and invasion could be regulated by miRNA through activation of tumor suppressor or inhibition of oncogene. While the altered expression of miRNA has been contributed to the aggressiveness of various types of cancer ${ }^{48}$.

Nevertheless, several miRNAs could target one sequence of mRNA, whereas one miRNA has numerous targets of mRNA ${ }^{49}$. In the human genome many miRNAs have been known to regulate thousands of genes. While miRNAs function as tumor suppressor or oncogene, its action could affect the tumorigenesis process as it disrupts the function of either oncogenic or suppressor genes ${ }^{50}$.

In 2008, miRNAs were first defined and after that more than 79 miRNAs are documented to be biomarker in many types of cancer. Therefore, miRNAs are considered an important prognostic biomarker. Also, miRNAs can be used as therapeutic targets in cancerous diseases treatment of ${ }^{51}$. 


\subsection{1. miRNAs Biogenesis:}

In the nucleus, RNA polymerase II makes transcription for a pri-miRNA precursor. Then, the endonucleases enzyme (DROSHA and DGCR8) deals with it to produce pre-miRNA sequence which is composed of $\sim 80-100$ nucleotides. The transportation of pre-miRNA from the nucleus to the cytoplasm is done by the aid of exportin-5. The dicer, cytoplasmic ribonucleases, cleaves the pre-miRNA into mature double stranded miRNA. The Argonaute (Ago) protein binds to mature miRNA creating RNAinduced silencing complex (RISC) that controls the mRNA translation ${ }^{52}$. Then the mature miRNA

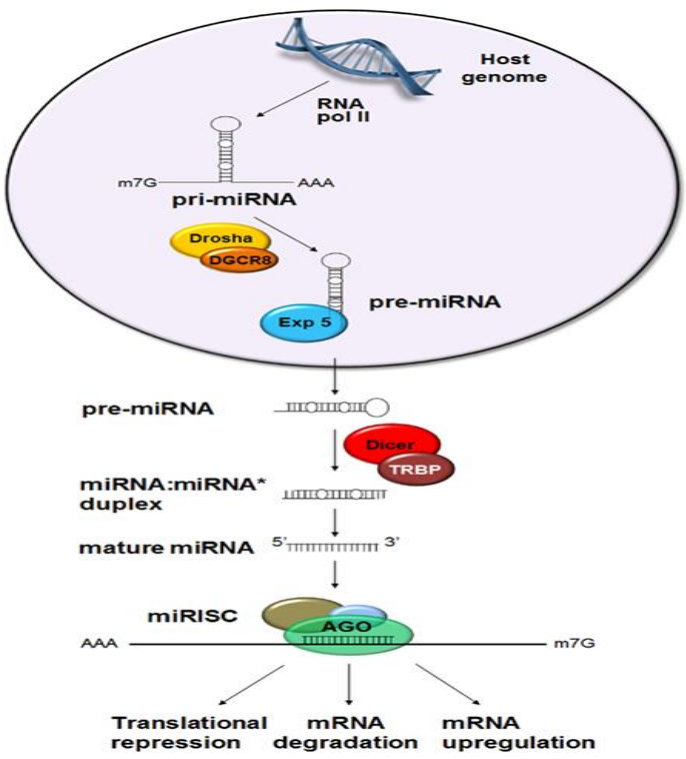

identifies its complementary sequences in 3'UTR of mRNA by seed region. Also, miRNA could attach to5'UTR or open reading frame (ORF) of mRNA ${ }^{53}$ (Figure 5).

Figure (5): MiRNA biogenesis and mechanism of action ${ }^{54}$.

\subsubsection{Functions of miRNA}

The miRNA can either cause degradation of mRNA or translational repression of protein. This action accomplished by two ways (1) perfect binding of miRISC to target perfectly complimentary mRNA which cause RNA degradation. (2) Imperfect joining of miRNA to 3' UTR or 5'UTR of partially complimentary mRNAs causes translational repression ${ }^{55}$. Accumulating evidence suggesting that miRNA has a positive effect on the translation of mRNA through increasing the transcription level ${ }^{56}$.

Some studies suggested that miRNAs are expressed only in cancerous tissue. The abnormal expression of miRNA during cancer permits the categorization of miRNA to tumor suppressor and oncogene. The oncogene related gene which promotes tumor invasion and proliferation could be targeted by a tumor suppressor miR such as miR-34 cluster (miR-34a, miR-34b and miR-34c). In cancer, miR-34 is downregulated due to the hypermethylation of its promoter region. The restoration of miR-34 hinders cancer progression ${ }^{57}$.

While miR-17-92 cluster functions as oncogenic miRNAs, and has a role in angiogenesis, metastasis, and proliferation in some diseases including solid cancer ${ }^{58}$. Nevertheless, some miRNAs have a dual role, this specific miRNA are concerned to be a tumor suppressor in some reports and oncogenic in others that may depend on the cancer type. The dual acting miR-125b which acts as oncogenic miR in hematological tumor and as tumor suppressor miR in solid cancer, this dual action could be clarified by the fact that several mRNA could be targeted by miR-125 59 . Also, the cancer immune system could be affected by the action of miRNA either as oncogenic or tumor suppressor ${ }^{60}$.

The dysregulation of miRNAs is related to tumor progression. There are numerous examples of miRNAs which are involved in tumorigenesis have been documented. The oncogenic miR-21 is contributed in BC epithelial to mesenchymal transition. Also, miR-9 is associated with a BCSC phenotype and EMT state. The suppression of miR200C seems to act in the tumorgenicity of BCSCs. While the reintroduction of miR-203 enhances the suppression of stem cell characteristics ${ }^{10}$. The ectopic expression of miR-150 encouraged growth and clonogenicity, and reduced apoptosis ${ }^{51}$.

\subsection{3. miRNA Expression Regulation and its Effect on Genes}

The fluctuation within the miRNA gene expression is concerned with the development of human cancers. The regulation of the expression of miRNA are accomplished by different ways ${ }^{61}$. Firstly, in the nucleus it is regulated by transcription of miRNA, while in cytoplasm it is controlled via processing of miRNA by DICER and DROSHA. Secondly, it is altered by RNA editing, uridylation, adenylation and RNA methylation ${ }^{53}$.

The epigenetic regulation disturbs not only the protein coding genes but also miRNAs. Affording to information from the genome sequence, the expression of miRNAs could be affected by its location. Some miRNAs are located in intron or exons and in either sense or antisense orientation. miRNA may be positioned in area subjected to deletion, amplification and mutation. The epigenetic silencing and the disturbance of TF could also induce the miRNA expression ${ }^{62}$.

DNA methylation plays a critical role in the alteration of miRNA expression. As discussed before, several miRNAs are located in $\mathrm{CpG}$ regions, so their expression could be affected by DNA methylation ${ }^{63}$. 
The alteration of the expression of miRNA not only happened because of its position in CGIs, but also its promoters contain TATA boxes, transcription factor II, and histone modification ${ }^{64}$. The transcription of miRNA promoter in the host gene occurs independently. The mature miRNA contains nucleosomes which helps in processing of pre$\operatorname{miRNA}^{65}$.

As mentioned above the regulation of the miRNA expression was done by the epigenetic modifications. There are some studies shown that there is a link between the epigenetic alteration of miRNA expression and the disease, including breast cancer, colorectal cancer, leukemia ${ }^{66}$.

The normal expression level of miRNA was found to be affected by the hyper/hypomethylation. This alteration of miRNA normal expression usually happens in human cancers. This alteration of miRNA expression includes down regulation of tumor suppressor miRNA by increasing methylation level and upregulation of oncogenic miRNA by decreasing methylation level ${ }^{67}$ as shown in (Figure 6).

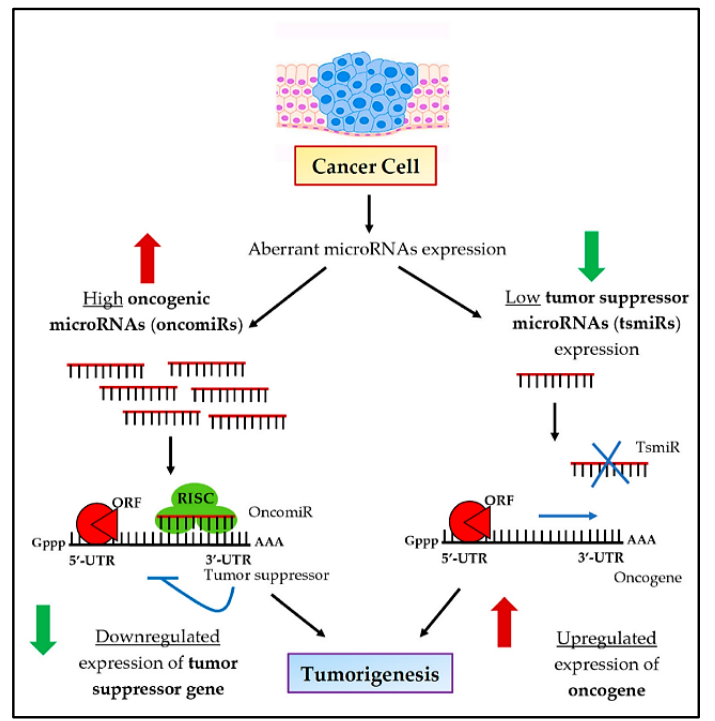

Figure (6): The epigenetic regulation of oncogenic and tumor suppressor microRNAs in cancer ${ }^{59}$.

The commonly informed epigenetically altered miRNAs in diverse types of cancer were miR-34 cluster, miR-124-121, miR-203, miR-127, miR199a, and others ${ }^{68}$. The downregulation of, for example, miR-203, miR-212, miR-200, and miR-124 family by abnormal hypermethylation of the promoter region will enhance the metastasis of cancer cells through EMT ${ }^{59}$.

\section{STEMNESS CHARACTER IN DIFFERENT CANCER TYPES:}

For more investigation to explore the changes in gene expression profile between TNBC and non
TNBC patients we analyzed the expression array of dataset (GSE27447) ${ }^{69}$ using the Gene Expression Omnibus (GEO). Heat map showed low expression of miR-203 and miR- 200C/141 and high expression of miR-150, CD44, DNMT1, DNMT3B and DNMT3A in TNBC in comparison to non TNBC. The heat map shows increase (red), decrease (blue), and means gene expression (white). The rows show individual tissue samples covering 14 non-triple negative breast tumors compared with 5 triple negative breast tumors.

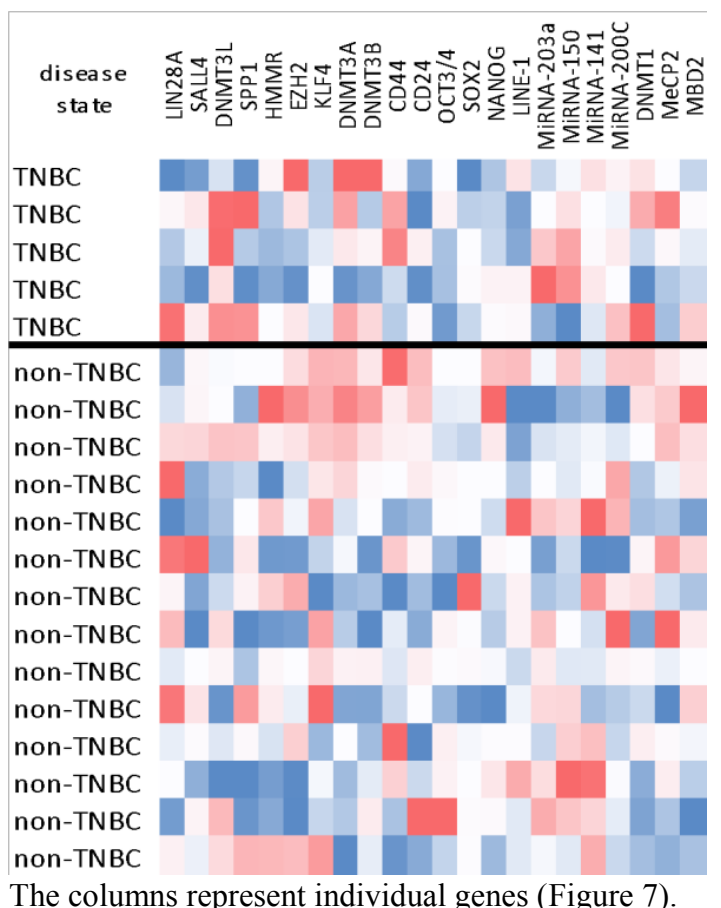

The columns represent individual genes (Figure 7).

Figure (7): Heat map shows genes expression profile in TNBC versus non TNBC patients of dataset GSE27447 ${ }^{69}$.

\section{Conclusion}

In this article, we discussed the association between epigenetic modification and miRNAs alteration in BCSCs. Abnormal methylation of DNA is considered as a major mechanism which disturb miRNA expression in cancer. DNA methylation controls by the hypermethylation or hypomethylation of the promoter-associated $\mathrm{CpG}$ islands regulates the miRNA expression including tumor-suppressor miRs and oncogenic miRs. Also, miRNAs could adjust DNA methylation by targeting DNMTs.

\section{Ethics Approval}

NA 


\section{References}

1. Kang L, Mao J, Tao Y, et al. Micro RNA-34a suppresses the breast cancer stem cell-like characteristics by downregulating Notch1 pathway. Cancer science 2015; 106: 700-708. doi: 10.1111/cas. 12656.

2. Liu S, Cong Y, Wang D, et al. Breast cancer stem cells transition between epithelial and mesenchymal states reflective of their normal counterparts. Stem Cell Reports 2014; 2: 78-91. doi: 10.1016/j.stemcr.2013.11.009.

3. Paholak HJ, Stevers NO, Chen H, et al. Elimination of epithelial-like and mesenchymal-like breast cancer stem cells to inhibit metastasis following nanoparticle-mediated photothermal therapy. Biomaterials 2016; 104: 145-157. https://doi.org/10.1016/j.biomaterials.2016.06.045

4. Biddle A, Gammon L, Liang X, et al. Phenotypic Plasticity Determines Cancer Stem Cell Therapeutic Resistance in Oral Squamous Cell Carcinoma. EBioMedicine 2016; 4: 138-145. doi: 10.1016/j.ebiom.2016.01.007.

5. Mishra VK, Johnsen S. Targeted therapy of epigenomic regulatory mechanisms controlling the epithelial to mesenchymal transition during tumor progression. Cell and tissue research; 356. Epub ahead of print 17 May 2014. DOI: 10.1007/s00441014-1912-y.

6. Sousa B, Ribeiro AS, Paredes J. Heterogeneity and plasticity of breast cancer stem cells. Stem Cells Heterogeneity in Cancer 2019; 83-103. doi: 10.1007/978-3-030-14366-4_5.

7. Chhabra R. miRNA and methylation: a multifaceted liaison. Chembiochem 2015; 16: 195-203. https://doi.org/10.1002/cbic.201402449

8. Yang F, Xu J, Tang L, et al. Breast cancer stem cell: the roles and therapeutic implications. Cellular and Molecular Life Sciences 2017; 74: 951-966. doi: 10.1007/s00018-016-2334-7.

9. Xu H, Wu K, Tian Y, et al. CD44 correlates with clinicopathological characteristics and is upregulated by EGFR in breast cancer. International journal of oncology 2016; 49: 1343-1350. doi: 10.3892/ijo.2016.3639.

10. Kwon MJ, Han J, Seo JH, et al. CD24 Overexpression Is Associated with Poor Prognosis in Luminal A and
Triple-Negative Breast Cancer. PLOS ONE 2015; 10: e0139112. doi: 10.1371/journal.pone.0139112.

11. Bozorgi A, Khazaei M, Khazaei MR. New Findings on Breast Cancer Stem Cells: A Review. Journal of Breast Cancer 2015; 18: 303-312. doi: 10.4048/jbc.2015.18.4.303.

12. Deng X, Apple S, Zhao H, et al. CD24 Expression and differential resistance to chemotherapy in triple-negative breast cancer. Oncotarget 2017; 8: 38294-38308. doi: 10.18632/oncotarget.16203.

13. Al-Hajj M, Wicha MS, Benito-Hernandez A, et al. Prospective identification of tumorigenic breast cancer cells. Proceedings of the National Academy of Sciences 2003; 100: 3983-3988. doi: 10.1073/pnas.0530291100.

14. Ahmed MA, Aleskandarany MA, Rakha EA, et al. A CD44-/CD24+ phenotype is a poor prognostic marker in early invasive breast cancer. Breast cancer research and treatment 2012; 133: 979-995. doi: 10.1007/s10549-011-1865-8.

15. Leis O, Eguiara A, Lopez-Arribillaga E, et al. Sox2 expression in breast tumours and activation in breast cancer stem cells. Oncogene 2012; 31: 1354-1365. doi: 10.1038/onc.2011.338.

16. Shen L, Huang X, Xie X, et al. High Expression of SOX2 and OCT4 Indicates Radiation Resistance and an Independent Negative Prognosis in Cervical Squamous Cell Carcinoma. J Histochem Cytochem 2014; 62: 499-509. doi: $10.1369 / 0022155414532654$.

17. Mashayekhi P, Noruzinia $\mathrm{M}$, Khodaverdi S. Deregulation of stemness-related genes in endometriotic mesenchymal stem cells: Further evidence for self-renewal/differentiation imbalance. Iranian Biomedical Journal 2020; 24: 328. doi: 10.29252/ibj.24.5.328

18. Roudi R, Barodabi M, Madjd Z, et al. Expression patterns and clinical significance of the potential cancer stem cell markers OCT4 and NANOG in colorectal cancer patients. Molecular \& Cellular Oncology 2020; $\quad 7: \quad 1788366$ https://doi.org/10.1080/23723556.2020.1788366

19. Huang C-F, Xu X-R, Wu T-F, et al. Correlation of ALDH 1, CD 44, OCT 4 and SOX 2 in tongue squamous cell carcinoma and their association with disease progression and prognosis. Journal of oral 
pathology \& medicine 2014; 43: 492-498. doi: 10.1111/jop. 12159 .

20. Amini S, Fathi F, Mobalegi J, et al. The expressions of stem cell markers: Oct4, Nanog, Sox2, nucleostemin, Bmi, Zfx, Tcl1, Tbx3, Dppa4, and Esrrb in bladder, colon, and prostate cancer, and certain cancer cell lines. Anatomy and Cell Biology 2014; 47: 1-11. doi: 10.5115/acb.2014.47.1.1

21. Dehghan Harati M, Rodemann HP, Toulany M. Nanog Signaling Mediates Radioresistance in ALDH-Positive Breast Cancer Cells. International Journal of Molecular Sciences 2019; 20: 1151. doi: 10.3390/ijms20051151.

22. Szklarczyk, D., Gable, A. L., Lyon, D., Junge, A., Wyder, S., Huerta-Cepas, J., ... \& Mering, C. V. STRING v11: protein-protein association networks with increased coverage, supporting functional discovery in genome-wide experimental datasets. Nucleic acids research 2019; 47(D1), D607-D613. doi: 10.1093/nar/gky1131

23. Alowaidi F, Hashimi SM, Nguyen $M$, et al. Investigating the role of CRIPTO-1 (TDGF-1) in glioblastoma multiforme U87 cell line. Journal of cellular biochemistry 2019; 120: 7412-7427. doi: $10.1002 /$ jcb.28015.

24. Klein RH, Tung P-Y, Somanath P, et al. Genomic functions of developmental pluripotency associated factor 4 (Dppa4) in pluripotent stem cells and cancer. Stem cell research 2018; 31: 83-94. doi: 10.1016/j.scr.2018.07.009.

25. Nagata T, Shimada Y, Sekine S, et al. Prognostic significance of NANOG and KLF4 for breast cancer. Breast cancer 2014; 21: 96-101. doi: 10.1007/s12282-012-0357-y.

26. Su R, Cao S, Ma J, et al. Knockdown of SOX2OT inhibits the malignant biological behaviors of glioblastoma stem cells via up-regulating the expression of miR-194-5p and miR-122. Molecular cancer 2017; 16: 1-22. doi: 10.1186/s12943-0170737-1.

27. Forghanifard MM, Kasebi P, Abbaszadegan MR. SOX2/SALL4 stemness axis modulates Notch signaling genes to maintain self-renewal capacity of esophageal squamous cell carcinoma. Molecular and Cellular Biochemistry 2020; 1-9. doi: 10.1007/s11010-020-03956-8.
28. Ma J, Qin L, Li X. Role of STAT3 signaling pathway in breast cancer. Cell Communication and Signaling 2020; 18: 1-13. doi: 10.1186/s12964020-0527-z.

29. Gaál, Z., \& Oláh, E. . Epigenetic regulatory mechanisms and their disorders in leukemia. Magyar onkologia,2014; 58(2), 99-107.

30. Abukiwan A, Berger MR. Epigenetics: dissecting gene expression alteration in PDAC. In: DNA Repair-An Update. IntechOpen, 2018.

31. Ren W, Gao L, Song J. Structural basis of DNMT1 and DNMT3A-mediated DNA methylation. Genes 2018; 9: 620. doi: 10.3390/genes9120620.

32. Benakanakere MR, Finoti L, Palioto DB, et al. Epigenetics, inflammation, and periodontal disease. Current Oral Health Reports 2019; 6: 37 46.

33. Teng H, Xue M, Liang J, et al. Inter-and intratumor DNA methylation heterogeneity associated with lymph node metastasis and prognosis of esophageal squamous cell carcinoma. Theranostics 2020; 10: 3035. DOI: 10.7150/thno.42559

34. Kribelbauer JF, Lu X-J, Rohs R, et al. Toward a mechanistic understanding of DNA methylation readout by transcription factors. Journal of molecular biology 2020; 432: 1801-1815.

35. Chiarella AM, Lu D, Hathaway NA. Epigenetic control of a local chromatin landscape. International journal of molecular sciences 2020; 21: 943 . DOI: 10.3390/ijms21030943

36. Pfeifer GP. Defining driver DNA methylation changes in human cancer. International journal of molecular sciences 2018; 19: 1166. DOI: $10.3390 /$ ijms 19041166

37. Dvorská D, Branỳ D, Nagy B, et al. Aberrant Methylation Status of Tumour Suppressor Genes in Ovarian Cancer Tissue and Paired Plasma Samples. International journal of molecular sciences 2019; 20: 4119 . DOI: $10.3390 /$ ijms20174119

38. Baba Y, Yagi $T$, Sawayama $H$, et al. Long Interspersed Element-1 Methylation Level as a Prognostic Biomarker in Gastrointestinal Cancers. DIG 2018; 97: 26-30. DOI: 10.1159/000484104

39. Briggs EM, Ha S, Mita P, et al. Long interspersed nuclear element-1 expression and 
retrotransposition in prostate cancer cells. Mobile DNA 2018; 9: 1-9. DOI: 10.1186/s13100-0170106-Z

40. Chen D, Wen X, Song YS, et al. Associations and prognostic implications of Eastern Cooperative Oncology Group performance status and tumoral LINE-1 methylation status in stage III colon cancer patients. Clinical Epigenetics 2016; 8: 36.

41. Glaich, O., Parikh, S., Bell, R. E., Mekahel, K., Donyo, M., Leader, Y. \& Levy, C. DNA methylation directs microRNA biogenesis in mammalian cells. Nature communications, 2019;10(1), 1-11. DOI: $10.1038 / \mathrm{s} 41467-019-13527-1$

42. Győrffy, B., Bottai, G., Fleischer, T., Munkácsy, G., Budczies, J., Paladini, L., \& Santarpia, L. Aberrant DNA methylation impacts gene expression and prognosis in breast cancer subtypes. International journal of cancer,2016; 138(1), 87-97. DOI: $10.1002 / \mathrm{ijc} .29684$

43. Khan AQ, Ahmed EI, Elareer NR, et al. Role of miRNA-Regulated Cancer Stem Cells in the Pathogenesis of Human Malignancies. Cells 2019; 8: 840. DOI: $10.3390 /$ cells 8080840

44. Lai S-C, Su Y-T, Chi C-C, et al. DNMT3b/OCT4 expression confers sorafenib resistance and poor prognosis of hepatocellular carcinoma through IL6/STAT3 regulation. Journal of Experimental \& Clinical Cancer Research 2019; 38: 474.

45. Sun X, Jiao X, Pestell TG, et al. MicroRNAs and cancer stem cells: the sword and the shield. Oncogene 2014; 33: 4967-4977. DOI: $10.1038 /$ onc. 2013.492

46. Saliminejad K, Khorshid HRK, Fard SS, et al. An overview of microRNAs: Biology, functions, therapeutics, and analysis methods. Journal of Cellular Physiology 2019; 234: 5451-5465. DOI: $10.1002 /$ jcp. 27486

47. Yin XZ, Zhao DM, Zhang GX, et al. Effect of miRNA-203 on cervical cancer cells and its underlying mechanism. Genet Mol Res; 15. DOI: $10.4238 /$ gmr. 15038680

48. Sun W, Zhang Z, Wang J, et al. MicroRNA-150 suppresses cell proliferation and metastasis in hepatocellular carcinoma by inhibiting the GAB1ERK axis. Oncotarget 2016; 7: 11595. DOI: 10.18632 /oncotarget.7292
49. Króliczewski J, Sobolewska A, Lejnowski D, et al. microRNA single polynucleotide polymorphism influences on microRNA biogenesis and mRNA target specificity. Gene 2018; 640: 66-72. DOI: $10.1016 /$ j.gene.2017.10.021

50. Qu Y, Pan S, Kang M, et al. MicroRNA-150 functions as a tumor suppressor in osteosarcoma by targeting IGF2BP1. Tumor Biology 2016; 37: 5275-5284. DOI: 10.1007/s13277-015-4389-8

51. Wang F, Ren X, Zhang X. Role of microRNA-150 in solid tumors. Oncology letters 2015; 10: 11-16. DOI: $10.3892 / \mathrm{ol} .2015 .3170$

52. Reddy KB. MicroRNA (miRNA) in cancer. Cancer Cell International 2015; 15: 38 DOI: $10.1186 / \mathrm{s} 12935-015-0185-1$

53. Treiber T, Treiber N, Meister G. Regulation of microRNA biogenesis and its crosstalk with other cellular pathways. Nature Reviews Molecular Cell Biology 2019; 20: 5-20. DOI: 10.1038/s41580018-0059-1

54. Asgari S. Role of MicroRNAs in Insect HostMicroorganism Interactions. Front Physiol; 2. Epub ahead of print 2011. DOI: 10.3389/fphys.2011.00048.

55. Michlewski G, Cáceres JF. Post-transcriptional control of miRNA biogenesis. RNA 2019; 25: 116. DOI: $10.1261 /$ rna.068692.118

56. O’Brien J, Hayder H, Zayed Y, et al. Overview of MicroRNA Biogenesis, Mechanisms of Actions, and Circulation. Front Endocrinol; 9. Epub ahead of print 2018. DOI: 10.3389/fendo.2018.00402.

57. Adams BD, Parsons C, Slack FJ. The tumorsuppressive and potential therapeutic functions of miR-34a in epithelial carcinomas. Expert Opinion on Therapeutic Targets 2016; 20: 737-753. DOI: $10.1517 / 14728222.2016 .1114102$

58. Liu X, Haniff HS, Childs-Disney JL, et al. Targeted Degradation of the Oncogenic MicroRNA 17-92 Cluster by Structure-Targeting Ligands. J Am Chem Soc 2020; 142: 6970-6982. DOI: $10.1021 /$ jacs.9b13159

59. Abdi J, Rastgoo N, Li L, et al. Role of tumor suppressor p53 and micro-RNA interplay in multiple myeloma pathogenesis. Journal of Hematology \& Oncology 2017; 10: 169. DOI: $10.1186 / \mathrm{s} 13045-017-0538-4$ 
60. Holubekova V, Mendelova A, Jasek K, et al. Epigenetic regulation by DNA methylation and miRNA molecules in cancer. Future Oncology 2017; 13: 2217-2222. DOI: 10.2217/fon-2017-0363

61. Sadri Nahand, J., Moghoofei, M., Salmaninejad, A., Bahmanpour, Z., Karimzadeh, M., Nasiri, M. \& Hamblin, M. R. Pathogenic role of exosomes and microRNAs in HPV-mediated inflammation and cervical cancer: a review. International journal of cancer,2020; 146(2), 305-320.

DOI: $10.1002 / \mathrm{ijc} .32688$

62. Valinezhad Orang A, Safaralizadeh R, KazemzadehBavili M. Mechanisms of miRNA-mediated gene regulation from common downregulation to mRNAspecific upregulation. International journal of genomics; 2014. DOI: 10.1155/2014/970607

63. Oltra, S. S., Peña-Chilet, M., Vidal-Tomas, V., Flower, K., Martinez, M. T., Alonso, E. \& Ribas, G. Methylation deregulation of miRNA promoters identifies miR124-2 as a survival biomarker in Breast Cancer in very young women. Scientific reports ,2018; 8(1), 1-12.

64. Park J-L, Lee Y-S, Kunkeaw N, et al. Epigenetic regulation of noncoding RNA transcription by mammalian RNA polymerase III. Epigenomics 2017; 9: 171-187. DOI: 10.2217/epi-2016-0108

65. Liu, B., Shyr, Y., Cai, J., \& Liu, Q. Interplay between miRNAs and host genes and their role in cancer. Briefings in functional genomics, 2019;18(4), 255-266. DOI: 10.1093/bfgp/elz002

66. Piletič K, Kunej T. MicroRNA epigenetic signatures in human disease. Archives of toxicology 2016; 90: 2405-2419. DOI: 10.1007/s00204-016-1815-7

67. Wang S, Wu W, Claret FX. Mutual regulation of microRNAs and DNA methylation in human cancers. Epigenetics 2017; 12: 187-197. DOI: $10.1080 / 15592294.2016 .1273308$

68. Strmsek Z, Kunej T. MicroRNA Silencing by DNA Methylation in Human Cancer: a Literature Analysis. Non-Coding RNA 2015; 1: 44-52. DOI: $10.3390 /$ ncrna1010044

69. Yang, L., Wu, X., Wang, Y., Zhang, K., Wu, J., Yuan, Y. C., \& Yen, Y. FZD7 has a critical role in cell proliferation in triple negative breast cancer. Oncogene 2011; 30(43), 4437-4446. doi:10.1038/onc. 